\title{
Suicidal Behavior in the First Wave of the COVID-19 Pandemic
}

\author{
Lucas Giner $^{1} \cdot$ Constanza Vera-Varela ${ }^{2} \cdot$ Diego de la Vega $^{1,3} \cdot$ Giovani M. Zelada $^{4} \cdot$ Julio A. Guija $^{1,5}$
}

Accepted: 15 December 2021 / Published online: 26 January 2022

(c) The Author(s), under exclusive licence to Springer Science+Business Media, LLC, part of Springer Nature 2022

\begin{abstract}
Purpose of Review Since the declaration of the COVID-19 pandemic, there have been numerous social changes to try to contain the spread of the disease. These sudden changes in daily life have also changed the way we relate to others, in addition to creating a climate of uncertainty and fear. Therefore, the objective of this review is to compile published data of the consequences of suicidal behavior in the first months from the onset of the pandemic.

Recent Findings The analysis reflects a concern about issues related to suicide since the beginning of the pandemic. A large number of online surveys have been released and have provided data on relatively large populations. The percentage of the population with suicidal ideation in that period seems to be approximately 5-15\%. Many studies associate suicidal ideation with being young, female, and presence of sleep problems. Surveys of healthcare workers do not seem to indicate a higher prevalence of suicidal ideation compared to the general population. The incidence of suicide attempts seen in emergency departments did not seem to change, while the number of visits for other issues, unrelated to suicide, did decrease. The few studies on completed suicide do not indicate an increase in incidence in these first 6 months since March 2020, when the WHO declared the start of the pandemic.

Summary It does not seem that there have been major changes in the figures related to suicidal behavior in the studies from the first wave of the COVID-19 pandemic, although it is still too early to know the consequences it will have long term. The social and economic damages resulting from the pandemic will certainly take a long time to recover.
\end{abstract}

Keywords Suicide $\cdot$ Suicidal behavior $\cdot$ Suicide attempt $\cdot$ Suicidal ideation · COVID-19 · Pandemic $\cdot$ Lockdown · Depression · Natural disasters · Psychological consequences

\section{Introduction}

On March 11, 2020, the World Health Organization (WHO) declared the spread of the Severe Acute Respiratory Syndrome Coronavirus 2 (SARS-CoV-2) virus, identified in

This article is part of the Topical Collection on Mood Disorders

Lucas Giner

lginer@us.es

1 Department of Psychiatry, Universidad de Sevilla, Seville, Spain

2 Servicio de Psiquiatría, Centro San Juan de Dios de Ciempozuelos, Madrid, Spain

3 Unidad de Hospitalización de Salud Mental. Unidad de Gestión Clínica de Salud Mental del Hospital Virgen Macarena. Servicio Andaluz de Salud, Seville, Spain

4 Internal Medicine/Psychiatry, University of Texas Southwestern Medical Center, Dallas, TX, USA

5 Instituto de Medicina Legal Y Ciencias Forenses de Sevilla, Seville, Spain late 2019 in China, as a new pandemic, causing the coronavirus disease 2019 (COVID-19). Its spread throughout the world has been very rapid and the intermediate and longterm social and disease consequences are not yet known. This pandemic has concerned many experts from the beginning about the possibility of an increase in suicide, calling the combination of factors "the perfect storm," since the pandemic itself and the political-health measures to con-

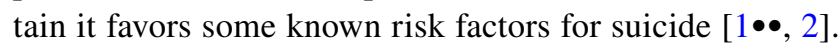
Thus, some problems exacerbated by the pandemic include increased economic stress, social isolation, barriers to mental health treatment, illness and medical problems, anxiety by media coverage, healthcare professional suicide rates, firearm sales, seasonal variation in rates, decreased access to community and religious support, increased alcohol and other substance misuses, unemployment, and financial loss, relationship problems (such as intimate partner violence) and loss $[1 \bullet \bullet, 2]$. At the beginning of the pandemic, suicides associated with the pandemic were published in the press, either from frontline health workers or those who had been 
infected [3, 4] and alerts appeared about the possible impact on mental health in general and increased risk of suicide, particularly in specific populations such as children and adolescents [5, 6], the elderly [7-9], health workers [10, 11••, 12] and in people with financial problems [10].

The relationship between suicides and natural disasters is known, thus women, adolescents, the elderly, those who suffered depression or post-traumatic stress disorder (PTSD), and those who had less social support or were without parents, have a greater risk of suicidal behavior [13]. Concerning other pandemics, such as the one suffered by Severe Acute Respiratory Syndrome (SARS) in 2002, 2003 and Middle East Respiratory Syndrome (MERS) in 2012 [14], some studies found a relationship between an increase in suicides associated with the respiratory pandemic of SARS and other viruses $[15,16]$, with a $31 \%$ increase in people over 65 years in Hong Kong 2003 at the peak of SARS, and the largest increase was primarily seen in older adults in the year following the pandemic [17]. Likewise, a higher risk of suicide was observed in the 12-year follow-up according to the adjusted hazard ratio depressive disorder 3.165 (95\% CI 2.465-4.077, $p<0.001$ ), anxiety disorder 3.172 (95\% CI 2.471-4.089, $p<0.001$ ), sleep disorder 2.411 (95\% CI 1.098-3.172, $p=0.001$ ), PTSD/ASD 60.360 (95\% CI 49.121-77.602, $p<0.001$ ) [18].

The consequences of the COVID-19 pandemic are in their early stages and we do not know how far they may go. Expert opinion, which is based on extrapolations with natural disasters (including pandemics), indicates that an increase in suicides is very likely after the crisis, while there may be a reduction during the crisis $[15,19 \bullet \bullet]$. For this reason, we would like to review the initial consequences of the COVID-19 pandemic on suicidal behavior.

\section{Method}

The authors conducted a search on PubMed since 5 years before until-2020/12/15 with the following query: "suicid*"[All Fields] AND ("sars cov 2"[MeSH Terms] OR "sars cov 2"[All Fields] OR "covid"[All Fields] OR "covid 19"[MeSH Terms] OR "covid 19"[All Fields]). A selection of studies best suited to our objective was performed, special focus was conducted on original studies with more than 20 cases that suicidal behavior (ideation, attempt, and completion) was analyzed. Moreover, reference lists from original studies were also screened.

\section{Results}

\section{Suicide Concern on the Internet}

The first published article that was retrieved by the prior research is a short article by Sher published in April 2020
[20] warning of the possible increased risk of suicide in relation to the COVID-19 pandemic. Media coverage of suicide cases has increased during the lockdown, at least in some countries [21]. These stories, without going into methods of transmission, generate a climate of concern when associating them to the pandemic. Through a direct assessment from surveys, an Australian study used qualitative analysis to assess the impact of the pandemic on family ( $n=2,130$ parents with children under 18 years of age), and six themes appeared, among them: "boredom, depression, and suicide: A spectrum of emotion" [22]. However, to determine the concern about certain issues in society, studies have been conducted to analyze the terms used on the internet, which not only indicate the number of times these terms are used, but also the association with other related terms and the evolution over several days.

Thus, in numerous studies on internet searches, it has been observed that at the beginning of the pandemic, there has been an increase in the search for terms related to work problems [23] and psychological terms such as loneliness, worry, and sadness, anxiety, panic attack or problems related to sleep [23-26]. The number of searches for insomnia was directly associated with the number of deaths in each country $(\rho=0.64, P=0.003)$, and the countries most affected in the first wave were the countries with the highest increase in searches insomnia (USA, Italy, Iran, and Spain) [27]. Other searches for terms such as stress, depression, suicide, and divorce, on the contrary, fell [23-27]. Another factor that could have influenced internet searches was the obligation of home confinement (stay-at-home orders). Thus, in the USA, the states with stay-at-home orders had a decrease in negative terms related to mental health (suicidal ideation, anxiety, negative thoughts, and sleep disturbances) compared to all other states. The terms that decreased the most were those related to suicidal ideation and anxiety [28].

In addition to internet searches, the analysis of web applications, such as Twitter or Reddit, also offers a social $\mathrm{X}$-ray of concerns or issues that stand out socially. Thus, data from Twitter in the USA indicated a considerable increase in posts related to mental health $(14 \%$ of crisismental health symptomatic expressions and 5\% of demonstrations of support), which attenuated as time passed, and that the authors associated to an adaptation to the "new normal" [29]. Analyzing the Reddit Mental Health dataset, which included 826961 posts (2018 to 2020), found that in the group of subjects with anxiety disorders, comments related to "economic stress," "isolation," and "home," had already increased by January. The groups with the most negative terms during the onset of the pandemic were attention-deficit/hyperactivity disorder, eating disorders, and anxiety disorders. The groups related to loneliness and suicidality doubled their activity and their activity 
was significantly closer to the borderline and PTSD groups [30].

\section{Effect on Suicidal Ideation}

The need to know all aspects related to and against the clock with COVID-19 has led to the development of various online surveys due to their easy distribution through emails and social networks. In the few studies on emergency care for suicidal ideation, it was found that care was either increased [31] or maintained [32,33]. In the case of the surveys that asked about suicidal ideation in relation to the pandemic among the general population, there are numerous studies in various countries, divided into specific populations, that have been repeated at different stages to see the evolution.

The studies with the general population began in China, as it is the country where the first SARS-CoV-2 infections were identified and from where the alarm was raised to the rest of the world, specifically in Wuhan. In February 2020, a survey was conducted with 1,397 participants from Wuhan and 2,794 controls from other Chinese cities, noting that subjects from Wuhan had more than double the risk of having anxiety and depression (adjusted $\mathrm{OR}=2.65,95 \%$ $\mathrm{CI}=2.14-3.29$ and adjusted $\mathrm{OR}=2.11,95 \% \mathrm{CI}=1.74-2.54$, respectively) and a slight increase in the OR for suicidal ideation $(\mathrm{OR}=1.60,95 \% \mathrm{CI}=1.28-2.02)$ [34]. Another survey in China (January-March 2020; $n=1172$ ) identified clinically significant symptoms of depression through the Patient Health Questionnaire (PHQ-9 $\geq 10$ ) in $18.8 \%$, and anxiety through the General Anxiety Disorder- 7 (GAD-7 $\geq 8$ ) in $13.3 \%$, and elevated suicide risk measured by the suicide subscale of the MINI at 2.8\%. In addition, clinical insomnia was found in $7.2 \%$ and clinical post-traumatic stress disorder symptoms in $7.0 \%$ [35]. Larger percentages were found in 19,515 pregnant women [36], where $44.6 \%$ had probable depression, $29.2 \%$ probable anxiety, $7.4 \%$ indicated having had suicidal ideation in the last 2 weeks and $0.7 \%$ had had it every day (PHQ-9, item 9), although the initial rate of suicidal ideation was $5.2 \%$ in the same population before the pandemic [37]. In a Hong Kong study on the use of a mask with 11,072 participants between 18 and 59 years old, they used the PHQ-9 to evaluate depression and suicidal ideation, finding that $15.5 \%$ had suicidal ideation for several days, $5.1 \%$ more than half of the days and $1.9 \%$ almost every day. With regards to depressive symptoms, $31.2 \%$ experienced mild symptoms, while $71.2 \%$ experienced moderate or severe depressive symptoms [38]. Lockdown itself seems to have a more important influence on mental health in the younger population, as indicated by the survey of students from 26 Chinese universities ( $n=24378$ ), whose risk of suffering from probable depression was $\mathrm{OR}=2.54$, while that of suicidal ideation or self-harm was $\mathrm{OR}=4.98$ compared to those without restrictions [39].
In other eastern countries, such as Taiwan $(n=1970)$, $10.8 \%$ reported having had suicidal ideas in the previous week (which were associated with having less perceived social support, being younger, having less frequency of handwashing, and worse physical health) and 55.8\% reported sleep problems [40]. In Bangladesh $(\mathrm{n}=10$ 067), reported depression in 33\% and suicidal ideation in 5\% (associated with being young, female, smoker, medical comorbidity, fear of COVID-19, and presence of sleep problems) [41]. In the same country, the prevalence of suicidal ideation was higher in the university population $(\mathrm{n}=3366)$ at $12.8 \%$, and was related to sleep problems, tobacco use, depression, anxiety, a history of suicidal ideation or suicide attempts, and a family history of suicidal behavior [42]. These figures were similar to those found in French university students $(n=69054)$, which reported suicidal ideas in $11.4 \%$, severe distress in $22.4 \%$, severe depression in $16.1 \%$, and high levels of anxiety in $27.5 \%$ [43]. In Libya $(n=2430)$, however, the figures for medical students were much higher, with suicidal ideation in $22.7 \%$, depressive symptoms in $88 \%$ (moderate or severe in $21.6 \%$ ), mild anxiety in $37.5 \%$, and moderate or severe anxiety in $27 \%$, although this study was conducted in a country in which there was a civil war [44].

With regards to Anglo-Saxon countries, in the UK $(n=3$ 077) they conducted a survey three times from the beginning of April to mid-May (the authors reported three waves), indicating a decrease in anxiety from $21 \%$ to $16.8 \%(\mathrm{OR}=0.82$ $[0.74-0.90],<0.0001)$, maintained levels of depressive symptoms $(26.1 \%-23.7 \%)$, while they indicate an increase in suicidal ideation in the period studied, from $8.2 \%$ to $9.8 \%(\mathrm{OR}=1.24[1.07-1.44], \mathrm{p}=0.005)$. The population group with the worst results were young people between 18 and 29 years old [45]. In Australia, despite having a minor impact of COVID-19 in this first wave, the percentages are similar in terms of depressive symptoms and much higher in terms of suicidal ideation. In a study of 13,829 subjects conducted in April 2020, 27.6\% had clinically significant symptoms of depression (PHQ-9 $\geq 10$ ), 21\% had clinically significant symptoms of anxiety (GAD-7 $\geq 10), 35.5 \%$ had ideas of death (PHQ-9, item 9) over several days in the last 2 weeks, $14.6 \%$ had more than half of the days, and $9.1 \%$ almost every day [46]. Figures comparable to those in another study over a longer time course ( 12 weeks; $n=5$ 455 ), indicated that both depressive symptoms (around $70 \%$ ) and suicidal ideation (around 30\%) have remained stable throughout the entire follow-up period, and suicidal intention/planning gradually decreased from $3.7 \%$ in the first 4 weeks to $2.1 \%$ in the last weeks, although this difference was not significant [47]. In the neighboring country of New Zealand $(n=2010)$, distress (moderate to severe) was observed in $30 \%$ and high anxiety in $16 \%$. With regards to suicidal behavior, $6 \%$ presented suicidal ideation, $2 \%$ planned suicide, and $2 \%$ attempted suicide (the worst results 
being related to younger age, loss or reduction of work, poor health, and a history of mental disorder) [48]. The largest samples were collected in the USA, and even with it being the beginning of the pandemic and with restrictive measures $(n=10625)$, a prevalence of suicidal ideation of $4.5 \%$ was found in the last month, with no differences in the subjects who were confined by the stay-at-home order, compared to those who weren't. Suicidal ideas in the last month were related to fights with a partner or serious legal problems [49]. In another study with a similar sample $(n=10368)$, they found higher numbers of subjects at risk of suicide, measured by the Suicide Behaviors Questionnaire-Revised (SBQ-R), at nearly $15 \%$, where the groups with the highest risk included ethnic minorities (Blacks, Native Americans, Hispanics), having children, singles, and the youngest age. Religion was identified as a protective factor, as were those with more social ties [50]. In a survey at the end of the first wave (end of June 2020, $n=5470$ ), $10.7 \%$ of the population had seriously thought about committing suicide in the last month, more than double than in 2018 (more frequent in women, young people of 18-24 years and 24-44 years, ethnic minorities, employed persons, and essential workers) [51]. In the American university population $(n=2031)$ they found higher figures than in the general population, with suicidal ideas in $18.04 \%$, moderate-severe depression (PHQ-9) in 48.14\%, and moderate-severe anxiety (GAD-7) in $38.48 \%$ [52].

Studies in other countries show similar figures, such as in Colombia $(\mathrm{n}=700)$, with $7.6 \%$ of subjects at high risk of suicide (associated with depression, perceived stress from COVID-19, and insomnia) [53]. In Czechoslovakia, when comparing two samples, one from $2017(\mathrm{n}=3000)$ and another during the pandemic $(\mathrm{n}=3000)$, they found that both the major depressive disorder and the risk of suicide tripled (3.96\% to $11.77 \%$ and $3.88 \%$ to $11.88 \%$, respectively), and anxiety disorders went from $7.79 \%$ to $12.84 \%$ [54]. On the other hand, a Greek study ( $\mathrm{n}=3$ 399), where COVID-19 had had little impact in the first wave, indicated that in $4.42 \%$ of the sample, suicidal ideas had increased and in $10.41 \%$ it had decreased [55].

A population that has been very important in its fight against the pandemic has been that of health personnel. Surveys of this population do not indicate a higher percentage of suicidal ideation than the general population, although they do in terms of other pathology. In China, a study of 4,692 first-line nurses indicated suicidal ideation in 6.5\%, while the presence of depressive and anxious symptoms was $9.4 \%$ and $8.1 \%$, respectively [56]. Identical figures regarding suicidal ideation were found in another study of 8,817 hospital workers $(6.5 \%$, with a higher risk associated with being a woman, ethnic minority, lower educational level, among others), while the figures for depression and anxiety were $30.2 \%$ (PHQ-9) and 20.7\% (GAD-7), respectively [57].
In direct comparison with the general population, the prevalence of suicide risk among medical staff $(n=606)$, measured by the suicidal module of the MINI, did not indicate differences in prevalence (13.0\% vs. $11.6 \%$, respectively). Additionally, there appeared to be an inverse relationship between years of experience and family income and suicide risk ( $p=0.03, p<0.001)$. However, a higher prevalence of depression measured by PHQ-9 (57.6\% vs. $47.6 \%$ ), anxiety by GAD-7 (45.4\% vs. $33.8 \%$ ), and insomnia using the Insomnia Severity Index (ISI) (32.0\% vs $25.1 \%$ ), was found in medical staff compared to the general population [58]. On the other hand, family members of first-line health workers had figures more similar to that of the general population, with $8.3 \%$ having suicidal or self-injurious thoughts. Additionally, clinically significant depressive symptoms were found in $12.2 \%$ of the sample, and anxiety problems in $49 \%$ [59]. In a survey in Bangladesh, which compared the general population $(n=2554)$ with healthcare workers $(n=3$ 388), similar figures were found in persistent suicidal ideation or suicide attempt concerning the pandemic in health workers $(6.1 \%)$ compared to the general population $(6 \%)$. In both health workers and the general population, suicidal ideation was more prevalent among women than among men (health workers: $8.1 \%$ vs $3.3 \% ; p<0.001$; general population: $8.5 \%$ vs $3.6 \%$; $p<0.001$ ). The risk factors were similar in the health workers and in the general population, which included, being a woman $(\mathrm{OR}=2.50$; 95\% CI $=1.83-3.42 ; p<0.001)$, being divorced $(\mathrm{OR}=3.77$; 95\% CI $=1.64-8.68 ; p<0.001)$, and not having children $(\mathrm{OR}=1.793 ; \mathrm{CI}=1.318-2.437 ; p<0.001)$ [60]. These figures are similar to those reported in other studies, where $5 \%$ of neurosurgeons (international sample $n=375$ ) [61] and of health workers $(n=1685)$ responded having suicidal ideation [59]. In this last study, it was also found that $29 \%$ had mild depressive symptoms while $17 \%$ had moderate or severe depressive symptoms [59].

\section{Mediators of Suicidal Ideation}

It has also been of particular interest to evaluate the psychological consequences of quarantine that could contribute to suicide risk and that, according to studies of previous disasters, can persist years later [62]. Among them, loneliness and PTSD stand out. In the USA, Killgore et al. studied loneliness at 3 weeks $(n=1013)$ [63••], in the first 3 months $(n=3$ 121) [64] and the progression at 6 months $(n=6186)$ [65], and found a correlation between feelings of loneliness (43\% of the sample) (measured by the UCLA Loneliness Scale-3) with depressive symptoms and suicidal thoughts (PHQ-9) in the first months (April $\rho=0.42$ ), May $\rho=0.40$, June $\rho=0.39$, all $p$ values $<0.00001$ ) and over 6 months $(\rho=0.40 ; p<0.00001)[64,65]$. In the progression, they 
observed that feelings of loneliness were greater in places where there had been restrictions (states with stay-at-home, shelter-in-place, or lockdown) and these feelings remained even though restrictions had been withdrawn [64, 65]. On the other hand, suicidal ideas increased every month in places with restrictions, and not in those without those restrictions [66]. The aftermath of the pandemic and lockdown has been related to an increase in the prevalence of PTSD, as some experts had already warned [67], and thus in the USA $14 \%$ of first-line health personnel $(n=1685)$, and $10.4 \%$ of relatives in China $(n=671)$ met criteria for PTSD screening within months of the onset of the pandemic [59, 68]. Once the lockdown ended, in China $(n=673) 10.8 \%$ of the workers met the criteria for PTSD, while $3.8 \%$ did so for anxiety, $3.7 \%$ for depression, and $2.3 \%$ for insomnia [69]. A third of the patients with previous psychiatric pathology met the PTSD criteria [70]. This is very important because the core symptomatology of PTSD related to COVID appears to be self-destructive/reckless behavior [71, 72]. Other identified mediators of suicidal ideation have been insomnia [73], psychological inflexibility [74], and the thwarted belongingness of the interpersonal theory of suicide [75].

\section{Incidence of Suicide Attempts}

Although cases of subjects with suicide attempts and debut psychotic symptoms with content closely related to the pandemic have been detected in Spain [76] and Germany [77], the objective would be to identify changes in the incidence of suicide attempts in general. Thus, in the USA, the incidence of suicide attempt in the last month at the beginning of the pandemic, was between 1.2 and $1.5 \%$ according to the survey $(n=10625)$, without the presence of restrictive measures related to life-threatening illness/injury of friend/ family [49]. With regards to suicide attempts treated in hospitals, the Psychiatric Liaison service experienced an increase in consultations for suicide attempts (OR 8.0, 95\% CI 2.03 to $31.57, p=0.003$ ), where the most common causes were related to relationship problems and loss of privacy during the lockdown [78]. In the surgical services, both in India and Scotland, the number of cases of suicide attempts by stabbing increased $[79,80]$.

In the ED, the number of visits generally saw a drastic decrease in different countries during lockdown [31, 33], even in more recently published [81]. However, the incidence of suicide attempts compared to previous periods seems to be similar [32,33] or lower, either in general [82] or only in the child-adolescent population [32]. Although it could be that there was an initial decrease and a subsequent increase, as indicated in an Irish study, where the initial decrease in attempts was $35 \%$ then increases to $104 \%$, with associated substance use [83].

\section{Completed Suicide Figures}

There are few studies in this regard, and it may be too early to measure the impact that the COVID-19 pandemic has on the number of suicides and we may have to wait months or years to assess it more accurately. One of the contributing factors to these predictions is based on the economic consequences, which are yet to be known. The calculations of suicide numbers related to a possible increase in unemployment have been the basis for the studies by McIntyre and Lee in the USA [84••] and in Canada [85], where they propose three scenarios according to the increase in unemployment: no change, moderate increase, and large increase. Thus, they calculated an increase in suicide rates by $1 \%$ when the unemployment rate increased by $1 \%$ between the years 1999 and 2018 (95\% CI 1.02-1.06, $p<0.0001$ ). If in 2018 the suicide rate was 14.8 per 100000 , then in 2021 it would increase to 15.7 per 100000 (95\% CI 15.3-16.1) if there was no increase in unemployment, or to 16.2 (95\% CI 15.7-16.8) if there is a moderate increase in unemployment, and to 17.4 (95\% CI 16.8-18.0) if unemployment rates increase extremely [84••]. Following the same analysis, the same authors calculate the possible increase in suicides in Canada, based on the rate of 10.9 suicides per 100000 in 2019 , and they calculated that the increase in suicides in 2021 would be 11.0 (95\% CI 10.3-11.8) per 100000 for an unchanged unemployment rate, or 11.5 (95\% CI 10.7-12.3) with a moderate increase in unemployment, and 13.6 (95\% CI 12.7-14.6) for an extreme increase in unemployment [85].

Despite how early it is to fully assess the consequences, we know that the situation has an influence on suicide, and at least the fear of the disease or the experience of lockdown have been identified as a precipitant of some reported cases of suicides [86-88], extended suicides [89] and suicide pacts [90]. To identify the precipitant of the disease, we need to know the last circumstances of the person, and the psychological autopsy is still considered the best method, even more so in the pandemic, where the collection of data from the medical history may be impaired due to the difficulty of accessibility to medical care [91]. The data available to us at this time is based on epidemiological data, no psychological autopsy studies have yet been published. Thus, in Japan, they found that during the summer months there was an increase in suicides in women, from July to September, between 20 and $30 \%$, compared to men and previous years [92]. In this same country, they found an increase in suicides in May in those under 20 years of age (incidence rate ratio $(\mathrm{IRR})=1.34,95 \% \mathrm{CI} 1.01$ to 1.78$)$, although the possible association with the closure of schools was ruled out [93]. Aside from these correlations, other studies did not find an increase in suicide figures in the first months of the pandemic. In a Norwegian study that collected official national figures during the first 3 months of the pandemic, 
they found a decrease in suicides, without demographic differences, compared to suicides in the previous 5 years [94]. A similar study in Peru found a decrease in the suicide rate (more evident in men) that increased just after lockdown was implemented, although without reaching pre-lockdown rates [95]. In local studies, there was no change in the number of suicides, and neither in Athens (Greece) during the first month of lockdown [96], nor in Queensland (Australia) in the months February to August 2020 [97]. Data from Connecticut (USA) indicate a $13 \%$ reduction in suicides during lockdown compared to the previous 5 years, although the non-white percentage was increased $(23 \%$ vs $11.7 \%$; $\mathrm{X}^{2}=7.13 ; p<0.01$ ), without a difference between sex and age [98].

\section{Suicide Risk in Subjects With Previous Psychiatric Pathology}

In patients with mental disorders, somatic health is, to a certain extent, at greater risk than in the general population due to various peculiarities. For example, those of the psychiatric patient (higher prevalence of medical comorbidities, impaired comprehension ability, behavioral alterations that, in some cases, may make preventive measures, treatment, and monitoring difficult) or of individuals in inpatient settings, as in the psychiatric wards, or areas in the hospital without providers trained in somatic problems or challenging socioeconomic conditions [99-101]. Studies on the impact on suicidal ideation in individuals with previous pathology are sparse; thus, in subjects with substance use, an increase in suicidal ideation and risky consumption was observed related to COVID-19, although it did not seem to increase the risk of overdose [102]. Older individuals (mean age 69) with depressive disorder in the first 2 months of the pandemic indicated that, despite having a worse quality of life, they had not stopped having social contact and were more concerned with contagion than isolation. The intensity of depressive symptoms, anxiety, and suicidal thoughts were similar to the pre-pandemic period [103].

\section{Suicidal Behavior in COVID Patients}

Although cases of first-episode psychosis and suicide attempt [104-106] or suicide have been described as a possible adverse effect in the use of Hydroxychloroquine for COVID-19 [107], the incidence of suicidal behavior in COVID + patients is anecdotal, as indicated by a study in NYC in the first wave March-April, where COVID + subjects compared to COVID- treated in the emergency room had a lower incidence of suicidal ideation $(16.7 \%$ vs $54.4 \%$ ), attempted suicide ( $0 \%$ vs $5.3 \%$ ), depression
(33.3\% vs. $49.1 \%)$ and major psychotic episode $(66.7 \%$ vs. $35.1 \%$ ) [32]. On the other hand, a retrospective study of 40,469 from a global health collaborative clinical held from January to June 2020 [108], described that $3.8 \%$ of COVID + patients presented with mood disorders (3.8\%) and only $0.2 \%$ had suicidal ideation. Regarding the psychological interventions carried out by psychiatrists via telephone in COVID + patients, it was reported that $9 \%$ had suicidal ideation, $18 \%$ had anxiety, $39 \%$ had depression, $30 \%$ had sleep problems, and a third of the patients noted improvement after initiating drug treatment [109]. In admitted or outpatient recovered patients $(n=284), 9.9 \%$ had one or more than one positive item of the short version of the MINI Suicidality Scale, only $2.8 \%$ presented a moderate risk, and none had high risk. According to this study, the highest risk would be associated with PTSD symptoms [72]. Some authors have suggested that SARS-CoV-2 virus may have a direct action on biological pathways related to suicidal behavior, which could also explain the increased risk of suicide observed in subjects with SARS $(18,110)$.

\section{Conclusions}

The COVID-19 pandemic is going to be a before and after in the history of health in the contemporary age. Like any natural disaster of this magnitude, it entails a series of consequences for humanity. The first and obvious, cases of morbidity and mortality from COVID, some of which include psychiatric disorders due to the direct action of SARS-CoV2. However, the great psychiatric blow comes indirectly, either by the loss of lives caused by the virus itself, by the deterioration of health care in general, or by the consequences secondary to the state of alarm and the strategies that have been implemented to reduce infection transmission and avoid a decline in health. Suicidal behavior will be among the consequences, no doubt. The true impact that COVID-19 has on suicide remains unknown, since this review only includes very preliminary studies, exclusively from the first wave, where there does not appear to have been significant changes in the number of people who have committed suicide or that suicide has been attempted. Nevertheless, the COVID-19 tsunami is still on land and the waters have not yet returned to the sea, making it difficult to quantify the damages and how long they will last, including the economic ones. We need time to understand the influence of the pandemic, as well as the mechanisms, in which suicidal behavior will be impacted.

Acknowledgments The editors would like to thank Dr. Philippe Courtet for taking the time to review this manuscript. 


\section{Compliance with Ethical Standards}

Conflict of Interest Lucas Giner, Constanza Vera-Varela, Giovani M. Zelada, and Julio A. Guija each declare no potential conflicts of interest. Diego de la Vega has received personal fees from Janssen.

Human and Animal Rights and Informed Consent This article does not contain any studies with human or animal subjects performed by any of the authors.

\section{Bibliography}

Papers of particular interest, published recently, have been highlighted as:

\section{$\bullet$ Of major importance}

1.• Reger MA, Stanley IH, Joiner TE. Suicide Mortality and Coronavirus Disease 2019-A Perfect Storm? JAMA Psychiat. 2020 (In this opinion piece, authors list the potential problems related to COVID-19 and the potential for increased risk of suicidal behavior. However, they propose a series of potentially implementable beneficial actions during the pandemic.).

2. Brown S, Schuman DL. Suicide in the Time of COVID-19: A Perfect Storm. J Rural Health. 2020.

3. Rahman A, Plummer V. COVID-19 related suicide among hospital nurses; case study evidence from worldwide media reports. Psychiatry Res. 2020;291:113-272.

4. Chen GF, Cheng YR, Ye L, Wang MW, Zhou MY, Zhang F, et al. Psychological support and the COVID-19 - A short report. Eur Rev Med Pharmacol Sci. 2020;24(15):8185-6.

5. Ramadhan MHA, Putri AK, Melinda D, Habibah U, Fajriyah UN, Aini S, et al. Children's Mental Health in the Time of COVID19: How Things Stand and the Aftermath. Malays J Med Sci. 2020;27(5):196-201.

6. Ye J. Pediatric Mental and Behavioral Health in the Period of Quarantine and Social Distancing With COVID-19. JMIR Pediatr Parent. 2020;3(2):e19867.

7. de Leo D, Trabucchi M. COVID-19 and the Fears of Italian Senior Citizens. Int J Environ Res Public Health. 2020;17(10).

8. Van Orden KA, Bower E, Lutz J, Silva C, Gallegos AM, Podgorski CA, et al. Strategies to Promote Social Connections Among Older Adults During 'Social Distancing' Restrictions. Am J Geriatr Psychiatry. 2020.

9. Badrfam R, Zandifar A. Asia and COVID-19; The need to continue mental health care to prevent the spread of suicide in the elderly. Asian J Psychiatr. 2020;54:102452.

10. Conejero I, Berrouiguet S, Ducasse D, Leboyer M, Jardon V, Olié E, et al. Suicidal behavior in light of COVID-19 outbreak: Clinical challenges and treatment perspectives. Encephale. 2020;46(3S):S66-72.

11.• Tracy DK, Tarn M, Eldridge R, Cooke J, Calder JDF, Greenberg N. What should be done to support the mental health of healthcare staff treating COVID-19 patients? Br J Psychiatry. 2020;217(4):537-9 (Authors describe the evidence for the prevention model based on primary, secondary, and tertiary intervention. Specifically, building supportive relationships improve "virtual" well-being support and favor rapid access to mental health, even for brief interventions.).

12. Neto MLR, Almeida HG, Esmeraldo JD, Nobre CB, Pinheiro WR, de Oliveira CRT, et al. When health professionals look death in the eye: the mental health of professionals who deal daily with the 2019 coronavirus outbreak. Psychiatry Res. 2020;288:112972.

13. Jafari H, Heidari M, Heidari S, Sayfouri N. Risk Factors for Suicidal Behaviours after Natural Disasters: A Systematic Review. Malays J Med Sci. 2020;27(3):20-33.

14. Yang Y, Peng F, Wang R, Yange GK, Jiang $T$, et al. The deadly coronaviruses. The 2003 SARS pandemic and the 2020 novel coronavirus epidemic in China. J Autoimmun. 2003;2020(109):102434.

15. Zortea TC, Brenna CTA, Joyce M, McClelland H, Tippett M, Tran MM, et al. The Impact of Infectious Disease-Related Public Health Emergencies on Suicide, Suicidal Behavior, and Suicidal Thoughts. Crisis. 2020:1-14.

16. Leaune E, Samuel M, Oh H, Poulet E, Brunelin J. Suicidal behaviors and ideation during emerging viral disease outbreaks before the COVID-19 pandemic: A systematic rapid review. Prev Med. 2020;141:106264.

17. Yip PS, Cheung YT, Chau PH, Law YW. The impact of epidemic outbreak: the case of severe acute respiratory syndrome (SARS) and suicide among older adults in Hong Kong. Crisis. 2010;31(2):86-92.

18. Tzeng NS, Chung CH, Chang CC, Chang HA, Kao YC, Chang SY, et al. What could we learn from SARS when facing the mental health issues related to the COVID-19 outbreak? A nationwide cohort study in Taiwan. Transl Psychiatry. 2020;10(1):339.

19.•• Wasserman D, Iosue M, Wuestefeld A, Carli V. Adaptation of evidence-based suicide prevention strategies during and after the COVID-19 pandemic. World Psychiatry. 2020;19(3):294-306 (This article discussed risk factors inherent to catastrophic situations, while taking into account the most vulnerable population affected by COVID, such as those with the worst socioeconomic situation or health workers, and the people who are restricted from having contact with loved ones, even at funerals.).

20. Sher L. An infectious disease epidemic and resilience to suicide. Aust N Z J Psychiatry. 2020;54(10):1040.

21. Pathare S, Vijayakumar L, Fernandes TN, Shastri M, Kapoor A, Pandit D, et al. Analysis of news media reports of suicides and attempted suicides during the COVID-19 lockdown in India. Int J Ment Health Syst. 2020;14(1):88.

22. Evans S, Mikocka-Walus A, Klas A, Olive L, Sciberras E, Karantzas G, et al. From ". Front Psychol. 2020;11:588667.

23. Knipe D, Evans H, Marchant A, Gunnell D, John A. Mapping population mental health concerns related to COVID-19 and the consequences of physical distancing: a Google trends analysis. Wellcome Open Res. 2020;5:82.

24. Brodeur A, Clark AE, Fleche S, Powdthavee N. COVID-19, lockdowns and well-being: Evidence from Google Trends. J Public Econ. 2021;193:104346.

25. Halford EA, Lake AM, Gould MS. Google searches for suicide and suicide risk factors in the early stages of the COVID-19 pandemic. PLoS ONE. 2020;15(7):e0236777.

26. Stijelja S, Mishara BL. COVID-19 and Psychological DistressChanges in Internet Searches for Mental Health Issues in New York During the Pandemic. JAMA Intern Med. 2020.

27. Lin YH, Chiang TW, Lin YL. Increased Internet Searches for Insomnia as an Indicator of Global Mental Health During the COVID-19 Pandemic: Multinational Longitudinal Study. J Med Internet Res. 2020;22(9):e22181.

28. Jacobson NC, Lekkas D, Price G, Heinz MV, Song M, O’Malley AJ, et al. Flattening the Mental Health Curve: COVID-19 Stayat-Home Orders Are Associated With Alterations in Mental Health Search Behavior in the United States. JMIR Ment Health. 2020;7(6):e19347. 
29. Saha K, Torous J, Caine ED, De Choudhury M. Psychosocial Effects of the COVID-19 Pandemic: Large-scale QuasiExperimental Study on Social Media. J Med Internet Res. 2020;22(11):e22600.

30. Low DM, Rumker L, Talkar T, Torous J, Cecchi G, Ghosh SS. Natural Language Processing Reveals Vulnerable Mental Health Support Groups and Heightened Health Anxiety on Reddit During COVID-19: Observational Study. J Med Internet Res. 2020;22(10):e22635.

31. Montalbani B, Bargagna P, Mastrangelo M, Sarubbi S, Imbastaro B, De Luca GP, et al. The COVID-19 Outbreak and Subjects With Mental Disorders Who Presented to an Italian Psychiatric Emergency Department. J Nerv Ment Dis. 2020.

32. Ferrando SJ, Klepacz L, Lynch S, Shahar S, Dornbush R, Smiley A, et al. Psychiatric emergencies during the height of the COVID19 pandemic in the suburban New York City area. J Psychiatr Res. 2020.

33. McAndrew J, O'Leary J, Cotter D, Cannon M, MacHale S, Murphy $\mathrm{KC}$, et al. Impact of initial COVID-19 restrictions on psychiatry presentations to the emergency department of a large academic teaching hospital. Ir J Psychol Med. 2020:1-8.

34. Wang Q, Feng H, Wang M, Xie Y, Hou B, Lu X, et al. Mental health and psychological responses during the coronavirus disease 2019 (COVID-19) epidemic: A comparison between Wuhan and other areas in China. Psychosom Med. 2020.

35. Ren Y, Qian W, Li Z, Liu Z, Zhou Y, Wang R, et al. Public mental health under the long-term influence of COVID-19 in China: Geographical and temporal distribution. J Affect Disord. 2020;277:893-900.

36. Yang X, Song B, Wu A, Mo PKH, Di JL, Wang Q, et al. Whether and how lockdown and mandatory quarantine regarding COVID19 may affect mental health among pregnant women in China: Potential social, cognitive, and eHealth-related mechanisms. J Med Internet Res. 2020.

37. Shi P, Ren H, Li H, Dai Q. Maternal depression and suicide at immediate prenatal and early postpartum periods and psychosocial risk factors. Psychiatry Res. 2018;261:298-306.

38. Bressington DT, Cheung TCC, Lam SC, Suen LKP, Fong TKH, Ho HSW, et al. Association Between Depression, Health Beliefs, and Face Mask Use During the COVID-19 Pandemic. Front Psychiatry. 2020;11:571179.

39. Xin M, Luo S, She R, Yu Y, Li L, Wang S, et al. Negative cognitive and psychological correlates of mandatory quarantine during the initial COVID-19 outbreak in China. Am Psychol. 2020;75(5):607-17.

40. Li DJ, Ko NY, Chen YL, Wang PW, Chang YP, Yen CF, et al. COVID-19-Related Factors Associated with Sleep Disturbance and Suicidal Thoughts among the Taiwanese Public: A Facebook Survey. Int J Environ Res Public Health. 2020;17(12).

41. Mamun MA, Sakib N, Gozal D, Bhuiyan AI, Hossain S, BodrudDoza M, et al. The COVID-19 pandemic and serious psychological consequences in Bangladesh: A population-based nationwide study. J Affect Disord. 2020;279:462-72.

42. Tasnim R, Islam MS, Sujan MSH, Sikder MT, Potenza MN. Suicidal ideation among Bangladeshi university students early during the COVID-19 pandemic: Prevalence estimates and correlates. Child Youth Serv Rev. 2020;119:105703.

43. Wathelet M, Duhem S, Vaiva G, Baubet T, Habran E, Veerapa E, et al. Factors Associated With Mental Health Disorders Among University Students in France Confined During the COVID-19 Pandemic. JAMA Netw Open. 2020;3(10):e2025591.

44. Elhadi M, Buzreg A, Bouhuwaish A, Khaled A, Alhadi A, Msherghi A, et al. Psychological Impact of the Civil War and COVID-19 on Libyan Medical Students: A Cross-Sectional Study. Front Psychol. 2020;11:570435.
45. O'Connor RC, Wetherall K, Cleare S, McClelland H, Melson AJ, Niedzwiedz CL, et al. Mental health and well-being during the COVID-19 pandemic: longitudinal analyses of adults in the UK COVID-19 Mental Health \& Wellbeing study. Br J Psychiatry. 2020:1-8.

46. Fisher JR, Tran TD, Hammarberg K, Sastry J, Nguyen H, Rowe H, et al. Mental health of people in Australia in the first month of COVID-19 restrictions: a national survey. Med J Aust. 2020;213(10):458-64.

47. Staples L, Nielssen O, Kayrouz R, Cross S, Karin E, Ryan K, et al. Internet Interv. 2020;22:100351.

48. Every-Palmer S, Jenkins M, Gendall P, Hoek J, Beaglehole $\mathrm{B}$, Bell C, et al. Psychological distress, anxiety, family violence, suicidality, and wellbeing in New Zealand during the COVID-19 lockdown: A cross-sectional study. PLoS ONE. 2020;15(11):e0241658.

49. Bryan CJ, Bryan AO, Baker JC. Associations among state-level physical distancing measures and suicidal thoughts and behaviors among U.S. adults during the early COVID-19 pandemic. Suicide Life Threat Behav. 2020:e12653.

50. Fitzpatrick KM, Harris C, Drawve G. How bad is it? Suicidality in the middle of the COVID-19 pandemic. Suicide Life Threat Behav. 2020.

51. Czeisler M, Lane RI, Petrosky E, Wiley JF, Christensen A, Njai $\mathrm{R}$, et al. Mental Health, Substance Use, and Suicidal Ideation During the COVID-19 Pandemic - United States, June 24-30, 2020. MMWR Morb Mortal Wkly Rep. 2020;69(32):1049-57.

52. Wang X, Hegde S, Son C, Keller B, Smith A, Sasangohar F. Investigating Mental Health of US College Students During the COVID-19 Pandemic: Cross-Sectional Survey Study. J Med Internet Res. 2020;22(9):e228-317.

53. Caballero-Domínguez CC, Jiménez-Villamizar MP, Campo-Arias A. Suicide risk during the lockdown due to coronavirus disease (COVID-19) in Colombia. Death Stud. 2020:1-6.

54. Winkler P, Formanek T, Mlada K, Kagstrom A, Mohrova Z, Mohr $\mathrm{P}$, et al. Increase in prevalence of current mental disorders in the context of COVID-19: analysis of repeated nationwide cross-sectional surveys. Epidemiol Psychiatr Sci. 2020;29:e173.

55. Fountoulakis KN, Apostolidou MK, Atsiova MB, Filippidou AK, Florou AK, Gousiou DS, et al. Self-reported changes in anxiety, depression and suicidality during the COVID-19 lockdown in Greece. J Affect Disord. 2020;279:624-9.

56. Hong S, Ai M, Xu X, Wang W, Chen J, Zhang Q, et al. Immediate psychological impact on nurses working at 42 governmentdesignated hospitals during COVID-19 outbreak in China: A cross-sectional study. Nurs Outlook. 2020.

57. Xiaoming X, Ming A, Su H, Wo W, Jianmei C, Qi Z, et al. The psychological status of 8817 hospital workers during COVID-19 Epidemic: A cross-sectional study in Chongqing. J Affect Disord. 2020;276:555-61.

58. Zhou Y, Wang W, Sun Y, Qian W, Liu Z, Wang R, et al. The prevalence and risk factors of psychological disturbances of frontline medical staff in china under the COVID-19 epidemic: Workload should be concerned. J Affect Disord. 2020;277:510-4.

59. Young KP, Kolcz DL, O'Sullivan DM, Ferrand J, Fried J, Robinson K. Health Care Workers' Mental Health and Quality of Life During COVID-19: Results From a Mid-Pandemic, National Survey. Psychiatr Serv. 2020:appips202000424.

60. Mamun MA, Akter T, Zohra F, Sakib N, Bhuiyan AKMI, Banik PC, et al. Prevalence and risk factors of COVID-19 suicidal behavior in Bangladeshi population: are healthcare professionals at greater risk? Heliyon. 2020;6(10):e05259.

61. Sharif S, Amin F, Hafiz M, Benzel E, Peev N, Dahlan RH, et al. COVID 19-Depression and Neurosurgeons. World Neurosurg. 2020;140:e401-10. 
62. Jurblum $\mathrm{M}, \mathrm{Ng} \mathrm{CH}$, Castle DJ. Psychological consequences of social isolation and quarantine: Issues related to COVID-19 restrictions. Aust J Gen Pract. 2020;49(12):778-83.

63.• Killgore WDS, Cloonan SA, Taylor EC, Dailey NS. Loneliness: A signature mental health concern in the era of COVID-19. Psychiatry Res. 2020;290:113117 (The authors analyzed the high incidence of having a sensation of loneliness in populations that were in a situation of legal lock-down, which prevented in-person social contact. In this sense, about half of the respondents had high levels of loneliness, which was also associated with depression and suicidal ideation.).

64. Killgore WDS, Cloonan SA, Taylor EC, Miller MA, Dailey NS. Three months of loneliness during the COVID-19 lockdown. Psychiatry Res. 2020;293:113392.

65. Killgore WDS, Cloonan SA, Taylor EC, Lucas DA, Dailey NS. Loneliness during the first half-year of COVID-19 Lockdowns. Psychiatry Res. 2020;294:113551.

66. Killgore WDS, Cloonan SA, Taylor EC, Allbright MC, Dailey NS. Trends in suicidal ideation over the first three months of COVID-19 lockdowns. Psychiatry Res. 2020;293:113390.

67. Dutheil F, Mondillon L, Navel V. PTSD as the second tsunami of the SARS-Cov-2 pandemic. Psychol Med. 2020:1-2.

68. Feng Z, Xu L, Cheng P, Zhang L, Li LJ, Li WH. The psychological impact of COVID-19 on the families of first-line rescuers. Indian J Psychiatry. 2020;62(Suppl 3):S438-44.

69. Tan W, Hao F, McIntyre RS, Jiang L, Jiang X, Zhang L, et al. Is returning to work during the COVID-19 pandemic stressful? A study on immediate mental health status and psychoneuroimmunity prevention measures of Chinese workforce. Brain Behav Immun. 2020;87:84-92.

70. Hao F, Tan W, Jiang L, Zhang L, Zhao X, Zou Y, et al. Do psychiatric patients experience more psychiatric symptoms during COVID-19 pandemic and lockdown? A case-control study with service and research implications for immunopsychiatry. Brain Behav Immun. 2020;87:100-6.

71. Jiang W, Ren Z, Yu L, Tan Y, Shi C. A Network Analysis of Posttraumatic Stress Disorder Symptoms and Correlates During the COVID-19 Pandemic. Front Psychiatry. 2020;11:568037.

72. Poyraz B, Poyraz CA, Olgun Y, Gürel Ö, Alkan S, Özdemir YE, et al. Psychiatric morbidity and protracted symptoms after COVID-19. Psychiatry Res. 2020;295:113604.

73. Killgore WDS, Cloonan SA, Taylor EC, Fernandez F, Grandner MA, Dailey NS. Suicidal ideation during the COVID-19 pandemic: The role of insomnia. Psychiatry Res. 2020;290:113134.

74. Crasta D, Daks JS, Rogge RD. Modeling suicide risk among parents during the COVID-19 pandemic: Psychological inflexibility exacerbates the impact of COVID-19 stressors on interpersonal risk factors for suicide. J Contextual Behav Sci. 2020;18:117-27.

75. Gratz KL, Tull MT, Richmond JR, Edmonds KA, Scamaldo KM, Rose JP. Thwarted belongingness and perceived burdensomeness explain the associations of COVID-19 social and economic consequences to suicide risk. Suicide Life Threat Behav. 2020.

76. Valdés-Florido MJ, López-Díaz Á, Palermo-Zeballos FJ, Martínez-Molina I, Martín-Gil VE, Crespo-Facorro B, et al. Reactive psychoses in the context of the COVID-19 pandemic: Clinical perspectives from a case series. Rev Psiquiatr Salud Ment. 2020;13(2):90-4.

77. Weise J, Schomerus G, Speerforck S. The SARS-CoV-2 Pandemic and an Attempted Suicide of a Patient with Delusional Disorder. Psychiatr Prax. 2020;47(4):218-20.

78. Jhanwar S, Krishnan V, Rohilla J. Consultation-Liaison Psychiatry During COVID-19 Lockdown: A Retrospective Chart Review. Cureus. 2020;12(10):e11048.

79. Acharya S, Dash RK, Das A, Hota M, Mohapatra C, Dash S. An Epidemiological Study of Cut Throat Injury During COVID-19
Pandemic in a Tertiary Care Centre. Indian J Otolaryngol Head Neck Surg. 2020:1-6.

80. Olding J, Zisman S, Olding C, Fan K. Penetrating trauma during a global pandemic: Changing patterns in interpersonal violence, self-harm and domestic violence in the Covid-19 outbreak. Surgeon. 2020

81. Olié E, Nogue E, Picot M, Courtet P. Hospitalizations for suicide attempt during the first COVID-19 lockdown in France. Acta Psychiatr Scand. 2021;143(6):535-6.

82. Dragovic M, Pascu V, Hall T, Ingram J, Waters F. Emergency department mental health presentations before and during the COVID-19 outbreak in Western Australia. Australas Psychiatry. 2020;28(6):627-31.

83. McIntyre A, Tong K, McMahon E, Doherty AM. COVID-19 and its effect on emergency presentations to a tertiary hospital with self-harm in Ireland. Ir J Psychol Med. 2020:1-7.

84.• McIntyre RS, Lee Y. Preventing suicide in the context of the COVID-19 pandemic. World Psychiatry. 2020;19(2):250-1 (In this study, a statistical analysis was conducted to look at the relationship of work problems in different scenarios according to greater or less optimism, the potential relationship with an increase during the COVID pandemic, and the possible increase in the number of deaths due to suicide.).

85. McIntyre RS, Lee Y. Projected increases in suicide in Canada as a consequence of COVID-19. Psychiatry Res. 2020;290:113104.

86. Mamun MA, Ullah I. COVID-19 suicides in Pakistan, dying off not COVID-19 fear but poverty? - The forthcoming economic challenges for a developing country. Brain Behav Immun. 2020;87:163-6.

87. Dsouza DD, Quadros S, Hyderabadwala ZJ, Mamun MA. Aggregated COVID-19 suicide incidences in India: Fear of COVID19 infection is the prominent causative factor. Psychiatry Res. 2020;290:113145.

88. Mengin A, Allé MC, Rolling J, Ligier F, Schroder C, Lalanne L, et al. Psychopathological consequences of confinement. Encephale. 2020;46(3S):S43-52.

89. Mamun MA, Bhuiyan AKMI, Manzar MD. The first COVID-19 infanticide-suicide case: Financial crisis and fear of COVID19 infection are the causative factors. Asian J Psychiatr. 2020;54:102365

90. Mamun MA. The first COVID-19 triadic (homicide!)-suicide pact: Do economic distress, disability, sickness, and treatment negligence matter? Perspect Psychiatr Care. 2020.

91. Aquila I, Sacco MA, Ricci C, Gratteri S, Ricci P. Quarantine of the Covid-19 pandemic in suicide: A psychological autopsy. Med Leg J. 2020;88(4):182-4.

92. Nomura S, Kawashima T, Yoneoka D, Tanoue Y, Eguchi A, Gilmour S, et al. Trends in suicide in Japan by gender during the COVID-19 pandemic, up to September 2020. Psychiatry Res. 2020;295:113622.

93. Isumi A, Doi S, Yamaoka Y, Takahashi K, Fujiwara T. Do suicide rates in children and adolescents change during school closure in Japan? The acute effect of the first wave of COVID-19 pandemic on child and adolescent mental health. Child Abuse Negl. 2020:104680.

94. Qin P, Mehlum L. National observation of death by suicide in the first 3 months under COVID-19 pandemic. Acta Psychiatr Scand. 2020.

95. Calderon-Anyosa RJC, Kaufman JS. Impact of COVID-19 lockdown policy on homicide, suicide, and motor vehicle deaths in Peru. Prev Med. 2020;143:106331.

96. Sakelliadis EI, Katsos KD, Zouzia EI, Spiliopoulou CA, Tsiodras S. Impact of Covid-19 lockdown on characteristics of autopsy cases in Greece. Comparison between 2019 and 2020. Forensic Sci Int. 2020;313:110365. 
97. Leske S, Kõlves K, Crompton D, Arensman E, de Leo D. Realtime suicide mortality data from police reports in Queensland, Australia, during the COVID-19 pandemic: an interrupted timeseries analysis. Lancet Psychiatry. 2020.

98. Mitchell TO, Li L. State-Level Data on Suicide Mortality During COVID-19 Quarantine: Early Evidence of a Disproportionate Impact on Racial Minorities. Psychiatry Res. 2020;295:113629.

99. Chevance A, Gourion D, Hoertel N, Llorca PM, Thomas P, Bocher R, et al. Ensuring mental health care during the SARSCoV-2 epidemic in France: A narrative review. Encephale. 2020;46(3):193-201.

100. Kuzman MR, Curkovic M, Wasserman D. Principles of mental health care during the COVID-19 pandemic. Eur Psychiatry. 2020;63(1):e45.

101. Warfield SC, Pack RP, Degenhardt L, Larney S, Bharat C, Ashrafioun $\mathrm{L}$, et al. The next wave? Mental health comorbidities and patients with substance use disorders in under-resourced and rural areas. J Subst Abuse Treat. 2020:108189.

102. Stack E, Leichtling G, Larsen JE, Gray M, Pope J, Leahy JM, et al. The Impacts of COVID-19 on Mental Health, Substance Use, and Overdose Concerns of People Who Use Drugs in Rural Communities. J Addict Med. 2020.

103. Hamm ME, Brown PJ, Karp JF, Lenard E, Cameron F, Dawdani A, et al. Experiences of American Older Adults with Pre-existing Depression During the Beginnings of the COVID-19 Pandemic: A Multicity. Mixed-Methods Study Am J Geriatr Psychiatry. 2020;28(9):924-32.
104. Gillett G, Jordan I. Severe psychiatric disturbance and attempted suicide in a patient with COVID-19 and no psychiatric history. BMJ Case Rep. 2020;13(10).

105. Chacko M, Job A, Caston F, George P, Yacoub A, Cáceda R. COVID-19-Induced Psychosis and Suicidal Behavior: Case Report. SN Compr Clin Med. 2020:1-5.

106. Zhang K, Shi Y, Liu H, Hashimoto K. A Case Report of Suicide Attempt Caused by Acute and Transient Psychotic Disorder during the COVID-19 Outbreak. Case Rep Psychiatry. 2020;2020:4320647.

107. Garcia P, Revet A, Yrondi A, Rousseau V, Degboe Y, Montastruc F. Psychiatric Disorders and Hydroxychloroquine for Coronavirus Disease 2019 (COVID-19): A VigiBase Study. Drug Saf. 2020;43(12):1315-22.

108. Nalleballe K, Reddy Onteddu S, Sharma R, Dandu V, Brown A, Jasti M, et al. Spectrum of neuropsychiatric manifestations in COVID-19. Brain Behav Immun. 2020;88:71-4.

109. Kim JW, Stewart R, Kang SJ, Jung SI, Kim SW, Kim JM. Telephone based Interventions for Psychological Problems in Hospital Isolated Patients with COVID-19. Clin Psychopharmacol Neurosci. 2020;18(4):616-20.

110. Conejero I, Nobile B, Olié E, Courtet P. How Does COVID19 Affect the Neurobiology of Suicide? Curr Psychiatry Rep. 2021;23(4):16.

Publisher's Note Springer Nature remains neutral with regard to jurisdictional claims in published maps and institutional affiliations. 